\title{
CAB-ROR2-ADC BA3021
}

National Cancer Institute

\section{Source}

National Cancer Institute. CAB-ROR2-ADC BA3021. NCI Thesaurus. Code C155812.

An antibody-drug conjug ate (ADC) composed of a conditionally active biologic (CAB) antibody against receptor tyrosine kinase-like orphan receptor 2 (ROR2) conjug ated to an as of yet undisclosed cytotoxic agent, with potential antineoplastic activity. Upon administration of CAB-ROR2-ADC BA3021, the anti-ROR2 antibody becomes activated through an as of yet not fully elucidated process, only under the unique microphysical conditions that are present in the tumor microenvironment (TME) as a result of the glycolytic metabolism of cancer cells and not in the microenvironment of normal, healthy tissues. Upon binding to ROR2-expressing cancer cells and internalization, the cytotoxic agent kills the cancer cells through an as of yet undisclosed mechanism of action (MoA). ROR2, highly expressed during embryonic development while only minimally expressed on certain normal, healthy cells, is involved in Wnt signal transduction and is overexpressed on certain cancer cells. It plays a key role in cancer cell proliferation, migration and invasion. High levels of ROR2 expression often correlates with poor prognosis. The CAB antibody allows for efficient binding to ROR2-expressing cancer cells only, thereby maximizing efficacy while minimizing toxicity by avoiding activation and thus binding of the antibody to normal, healthy ROR2-expressing cells under normal conditions. 